\title{
BSS with Heterogeneous Sources
}

\author{
Vincent MARIE \\ NEXEYA Test Range Product Manager \\ Dedicated to the memory of Julien MALIVERNEY
}

\begin{abstract}
Telemetry ground stations have to ensure missions with always growing complexity (reception from various data provider, increase of data load, distribution on several sites) making them more and more expensive to maintain. Their refurbishment is usually carried out by successive iterations in order to reduce costs and secure the integration of new hardware and software bricks. One major consequence is the appearance of heterogeneous data formats representing the same telemetry data $(\mathrm{CH} 10$ packed, unpacked, PCM Data + Clock, raw PCM over IP, TMolP, Hardware proprietary format, etc.). This article describes architectures and methods we implemented in order to assure the most efficient BSS / BSC processing of those heterogeneous data formats with various data quality factors (as DQM, DQE), time desynchronization of the sources, allowing to provide, as a selection or as a combination of telemetry data, the best telemetry stream to the end user.
\end{abstract}

Key words: Telemetry, Best Source Selector, Best Source Combination, Ethernet, Time Synchronization, Software Frame Sync

\section{Introduction}

For more than a decade, telemetry data formats have evolved as much on board as on ground installation. New data encapsulation formats have emerged $(\mathrm{CH} 10, \mathrm{CH} 7$, TMoIP, IENA, AFDX, Hardware proprietary format, etc.); transmission protocols have also evolved to come closer to standard network formats (TCP / IP, UDP / IP). Telemetry systems are complex installations which must be able to adapt to these changes without compromising their general architecture.

Telemetry Range with distributed architecture are connected through Ethernet network and Analog signals have been reduced to the minimum length. Ethernet assets are obvious: Standard cable connectors, worldwide protocol for data transfer from \& to anywhere. That has been a great opportunity to reduce the cost of the telemetry installation with distributed architecture.

Large Telemetry Ranges with several remote sites have to proceed to Best Source Selection (or Best Source Combination) to ensure to get in real time the best telemetry stream without interruption and at any position of the target to track. To do this, it is required to concentrate all telemetry streams to the Telemetry Data Centre and this whatever the data format proposed by the hardware. Nowadays, installations evolve from Analog signal transmission to Ethernet transmission through mux / demux equipment. However, these evolutions can be expensive, they must therefore be able to be carried out iteratively, to reduce the cost but also to ensure a simpler validation of the modifications as well as to preserve the operational continuity of the installations. Nevertheless, BSS/BSC has still to be done from any Antenna on the range. Currently, Most of the Telemetry Ground Station equipment (receivers, decom stations) provide Ethernet outputs. However, the Ethernet protocol is not always following a standard and can be based on a proprietary Ethernet format.

BSS/BSC, which has to manage all these streams (Ethernet, Data+Clock, etc.), needs first to standardize the inputs in a common data format before proceeding to the Best Source Selection or Best Source Combination Algorithm.

This paper presents:

- The most common PCM formats and quality factors

- The different packet transmission methods

- The BSS/BSC architecture that NEXEYA experimented 
- Some practical cases

A conclusion based on the case study results

\section{Most common PCM format and quality factors PCM CH4 Streams Description:}

$\mathrm{PCM} \mathrm{CH} 4$ are defined as follow:

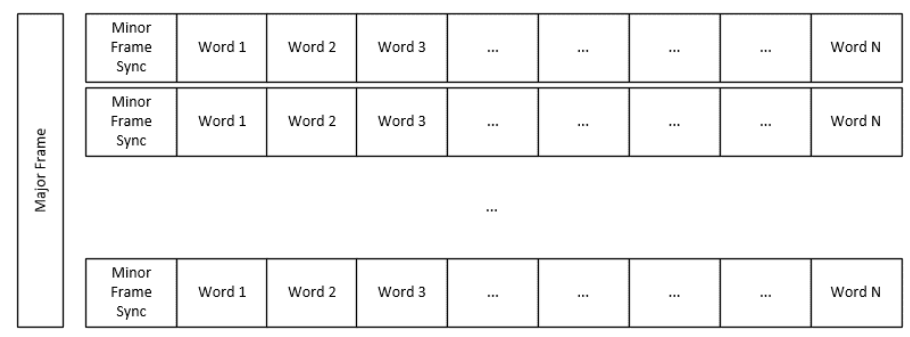

Figure 1: $\mathrm{PCM} \mathrm{CH4} \mathrm{format}$

\section{PCM Packet Timestamp}

PCM packets can be dated in several ways:

- $\quad$ Packets are dated when the frame is transmitted. For example, each short cycle contains reserved words containing its timestamp

- Packet are timestamped by reception hardware. The reception equipment adds a proprietary dating header. This format may differ from one supplier to another.

Quality Factors

Generally, raw PCM frames do not contain any quality factor. It is however possible to calculate, using to the content of the minor frame, some quality factors using the following information:

- $\quad$ Number of bits in error on the synchronization word

- SFID value

- Parity errors on PCM words

- Calculation and verification of a CRC present in the cycle

\section{CH10 PCM Streams Description:}

PCM Packet in $\mathrm{CH} 10$ streams are defined as follow:

\begin{tabular}{|c|c|c|c|c|c|c|c|c|c|}
\hline $\begin{array}{c}\text { Packet } \\
\text { Header }\end{array}$ & $\begin{array}{c}\text { Channel } \\
\text { Specific Data }\end{array}$ & $\begin{array}{c}\text { Intra-Packet } \\
\text { Time Stamp } \\
\text { (opt) }\end{array}$ & $\begin{array}{c}\text { Intra-Packet } \\
\text { Data Header } \\
\text { (opt) }\end{array}$ & $\begin{array}{c}\text { Minor Frame } \\
\text { Data }\end{array}$ & $\begin{array}{c}\text { Channel } \\
\text { Specific Data }\end{array}$ & $\begin{array}{c}\text { Intra-Packet } \\
\text { Time Stamp } \\
\text { (opt) }\end{array}$ & $\begin{array}{c}\text { Intra-Packet } \\
\text { Data Header } \\
\text { (opt) }\end{array}$ & $\begin{array}{c}\text { Minor Frame } \\
\text { Data }\end{array}$ & $\begin{array}{c}\text { Packet } \\
\text { Trailer }\end{array}$ \\
\hline
\end{tabular}

Figure 2: PCM Packet in $\mathrm{CH} 10$ format

Three different transmission modes are available in $\mathrm{CH} 10$ mode

- Pack

- Unpacked

- Throughput

\section{PCM Packet Timestamp}

The PCM packets are timestamped using a 48 bits counter (100 ns precision)

The PCM packets are timestamped using the optional header "Intra Packet Time Stamp". If the header is missing, the packet is timestamped using the last received "Time Packet". If both packets are present, a correlation can be made to ensure data synchronization.

\section{Quality Factors}

Generally, PCM packets in $\mathrm{CH} 10$ streams do not contain any quality factor. It is however possible to calculate, using to the content of the minor frame, some quality factors using the following information: 
- Number of bits in error on the synchronization word

- SFID value

- Parity errors on PCM words

- Calculation and verification of a CRC present in the cycle

\section{CH7 PCM Streams Description:}

$\mathrm{CH} 7$ is an encapsulation of $\mathrm{CH} 10$ streams and proprietary network streams. The PCM data is generally sent in $\mathrm{CH} 10$ packets

TMoIP (IRIG 218-10) Streams Description:

TMoIP (IRIG 218-10) are defined as follow:

\begin{tabular}{|c|c|}
\hline $\begin{array}{c}\text { TMolP } \\
\text { Control } \\
\text { Word }\end{array}$ & Raw Packet Payload \\
\hline
\end{tabular}

Figure 3: TMolP Packet Format

\section{Packet Timestamp}

The TMoIP (IRIG 218-10) packet format does not contain any clock information that would be useful in reconstructing the PCM output at the receiving device. The standard makes a reference to using the IETF RFC 1889 Real Time Protocol (RTP) to provide clock recovery support for TMoIP (IRIG 218-10). The required RTP header fields are shown in the following figure and the IRIG 218-10 packet is placed in the payload of the RTP packet.

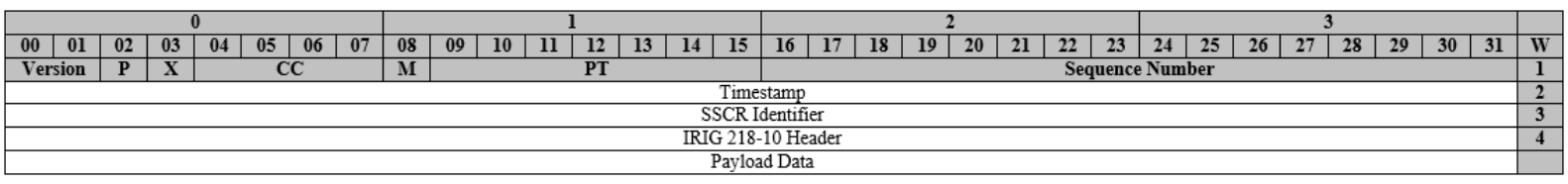

Figure 4 : RTP Header for IRIG 218-10 timestamp

\section{Quality Factors}

The TMoIP (IRIG 218-10) implements Data Quality Encapsulation (DQE) standard and Data Quality Metrics (DQM) standard as described in IRIG 106 Chapter 2 Appendix G9.

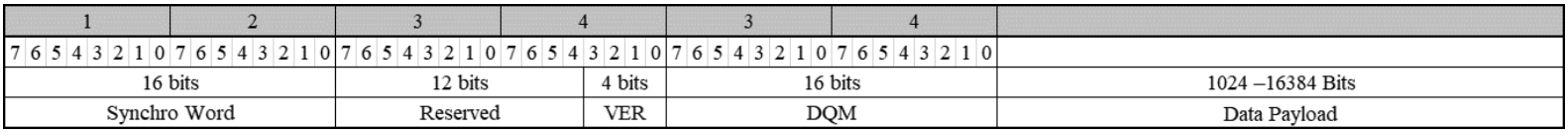

Figure 5: DQE format

The data payload is prefixed by a header including a synchronization word (0xFAC4), a version and the Data Quality Metrics (DQM).

\section{TMoIP (IRIG 218-20) Streams Description:}

TMoIP (IRIG 218-20) are defined as follow:

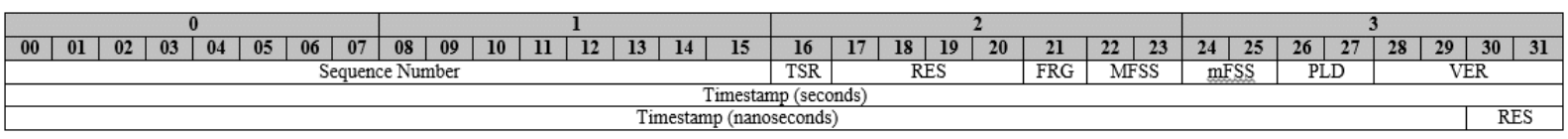

Figure 6 : IRIG 218-20 Header Format

Packet Timestamp

TMolP (IRIG 218-20) are timestamped using a 64-bit timestamp in PTP format:

- 32-bit seconds field

- 30-bit nanoseconds field

- Timestamps are relative to 00:00 01 Jan 1970

$\underline{\text { Quality Factors }}$ 
The TMoIP (IRIG 218-10) implements DQE standard and DQM standard as described in IRIG 106 Chapter 2 Appendix G9.

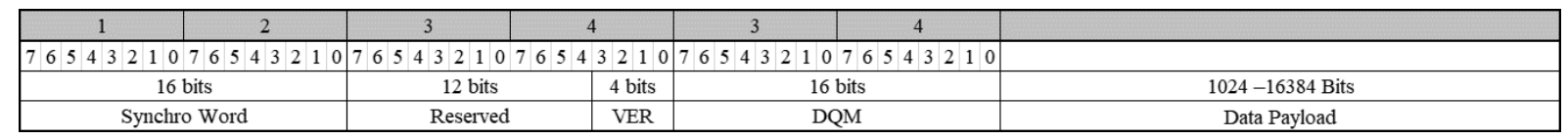

Figure 7: $D Q E$ format

The data payload is prefixed by a header including a synchronization word (0xFAC4), a version and the Data Quality Metrics (DQM).

\section{PCM Ethernet Streams Description:}

Ethernet is a wide world with open protocol and data format. This is the same with Ethernet PCM streams, different data normalization are available. This section introduces the Major cases.

Here is the standard format of a PCM Ethernet Message:

\begin{tabular}{|c|c|c|}
\hline $\begin{array}{c}\text { IP } \\
\text { Header }\end{array}$ & $\begin{array}{c}\text { UDP } \\
\text { Header }\end{array}$ & UDP Payload \\
\hline
\end{tabular}

Figure 8: Example of Ethernet UDP message

IP/UDP header will not be explained here; only payload has interest. It is generally formatted according to manufacturer's Telemetry receiver, or following a standard (UDP Ch10, Ch7, iNet, PCM over Ethernet...).

Generally, the UDP payload consists in the following data:

\begin{tabular}{|c|c|c|c|c|c|}
\hline Propriatany & $\begin{array}{l}\text { Time } \\
\text { Stamp }\end{array}$ & propratan & $\begin{array}{l}\text { Quality } \\
\text { Factors }\end{array}$ & poputary & PCM Stream \\
\hline
\end{tabular}

Figure 9: Example of UDP/IP payload

Common PCM Information:

The packet Timestamp

The quality factors (if provided)

The PCM stream

Packet Timestamp

Packet Timestamp is mandatory as Ethernet is non-deterministic protocol. Therefore, The Ethernet source of the PCM stream needs to have timestamped the packet before sending it on the Network.

\section{Quality Factors}

Quality factors also depends on the Ethernet Source. These information depend on the ability of the receiver. Generally, we have to deal with AGC, Eb/NO, and Lock Status.

Finally, Each Manufacturer sends the PCM data stream in a dedicated format with its own positioning \& its own format (integer, float, double format...). It is then necessary to apply the right process to extract all the required information.

\section{PCM Packet Data Transmission:}

Three possibilities of PCM stream transmission are possible:

- Unpacked

- Packed

- Throughput

Unpacked mode is a synchronized data flow with PCM word set on a 16-bit or 32-bit word container with additional padding. Therefore, to manage this stream it is necessary to know the word container size and the alignment (right or left). 


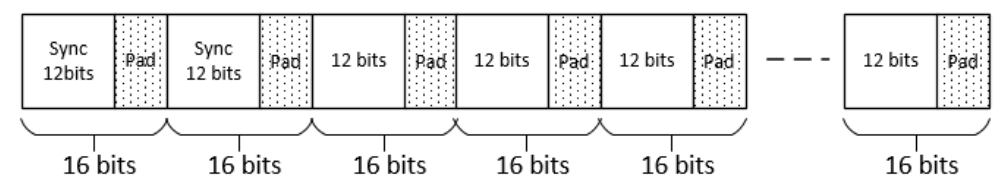

Figure 10: PCM Unpacked format

PCM word on 16-bit word container

Packed mode is a synchronized minor frame put on a 16-bit or 32-bit payload size. So Additional bits are added at the end to complete the minor frame on a 16-bit or 32-bit full size frame.

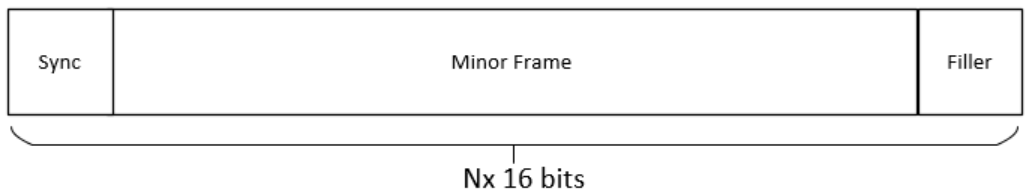

Figure 11 PCM Packet format

Throughput mode is used by telemetry receivers, which are not composed of a Frame Sync. So digital data are provided directly from the Bitsync Output and the stream is a raw data stream which could still be encrypted and not synchronized.

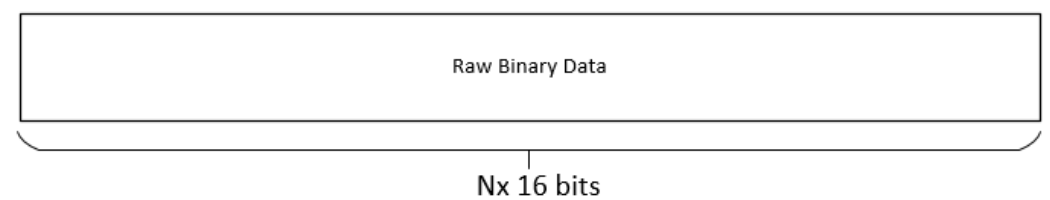

Figure 12: PCM Throughput format

These three formats can be possible in the Ethernet output stream of a TM station and in order to compare the contents, it is first required to standardize the format before proceeding to BSS.

\section{BSS / BSC Architecture}

With these PCM stream formats, it is now possible to determine the different required tasks to define a BSS / BSC with an architecture able to manage these heterogeneous streams. Regarding the previous chapter an architecture based on three layers is adequate:

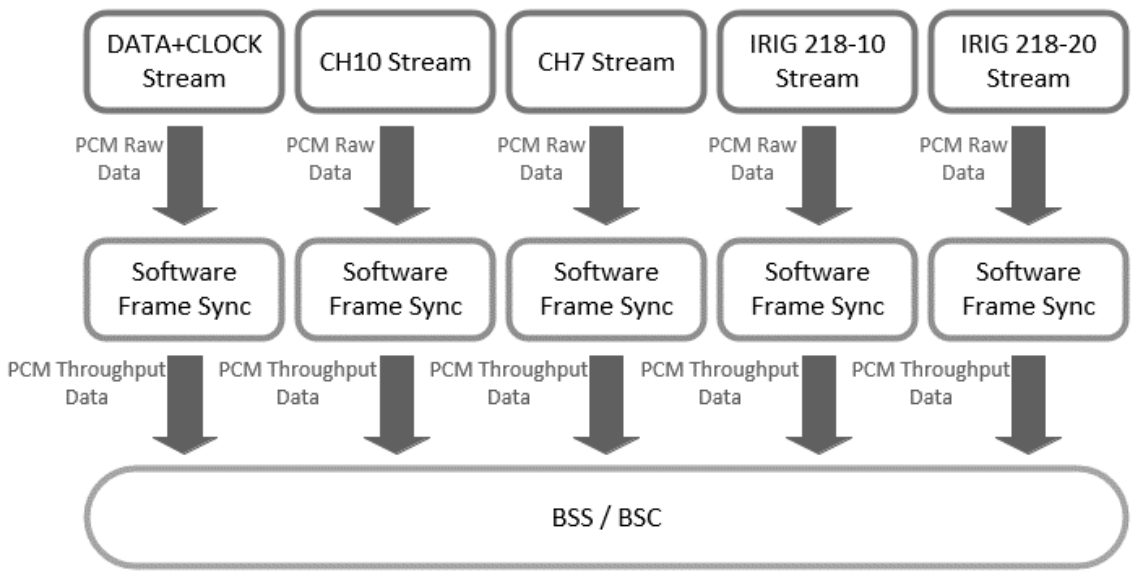

Figure 13: BSS / BSC Architecture

Proprietary Protocol layer is in charge of extracting the required information for the BSS from specific data format:

- Timestamp

- PCM stream

- Quality factor (if provided) 
This layer will be specific depending on the provider (manufacturer) of the telemetry source. There will be as much Proprietary Protocol layer module as manufacturers.

Software Frame Sync layer will have three goals:

- Proceed to the frame sync on throughput data

- Reformat extracted data (unpacked, packed, throughput) for suitable comparison in BSS

- Compute the quality factors based on the minor frame data (parity, synchronization word error bits, etc.).

Using the Software Frame Sync will sort the data in a common format to make data easy to compare for BSS.

Parameters input of this frame sync are

- $\quad$ The PCM format (FS size, MF size, mf size, word size etc...)

- The Word mapping (Word container size: 16 or 32 bits, MSB or LSB...)

- PCM payload buffer

- Timestamp of the buffer

- Format of the quality factors provided (DQM, EB/NO, Parity, Synchronization word bit errors, etc.)

From these inputs, the soft frame sync will provide a timestamped and qualified standardized output.

The interest of the Software Frame Sync is that any kind of data format can be managed: Throughput data, PCM words from 3 bits to 32 bits, swapped or not, MSB or LSB...

Finally, the output is always in the same format: MSB PCM throughput data.

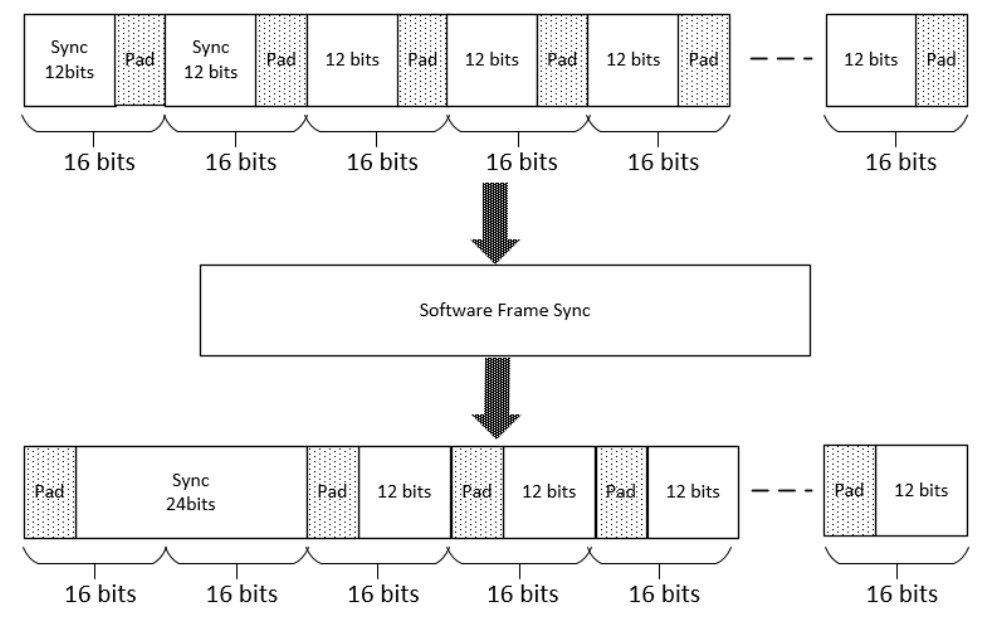

Figure 14: Reformatting data example

Figure above shows the reformatting of a 12 bits PCM word packed stream. The output holds the same data but in a different way that will be comparable with the other sources.

Once this part is complete, the BSS will always work on standardized data streams.

BSS can then be applied on all the sources received, as they are now comparable. Additional Quality factors extracted from the proprietary layer can help to the decision following the criteria and the weighting associated to these factors. Each minor frame is timestamped and with SFID information, it is possible to time align the different sources and proceed to BSS algorithm.

Figure below shows an example of a PCM stream comparison from three heterogeneous sources. 


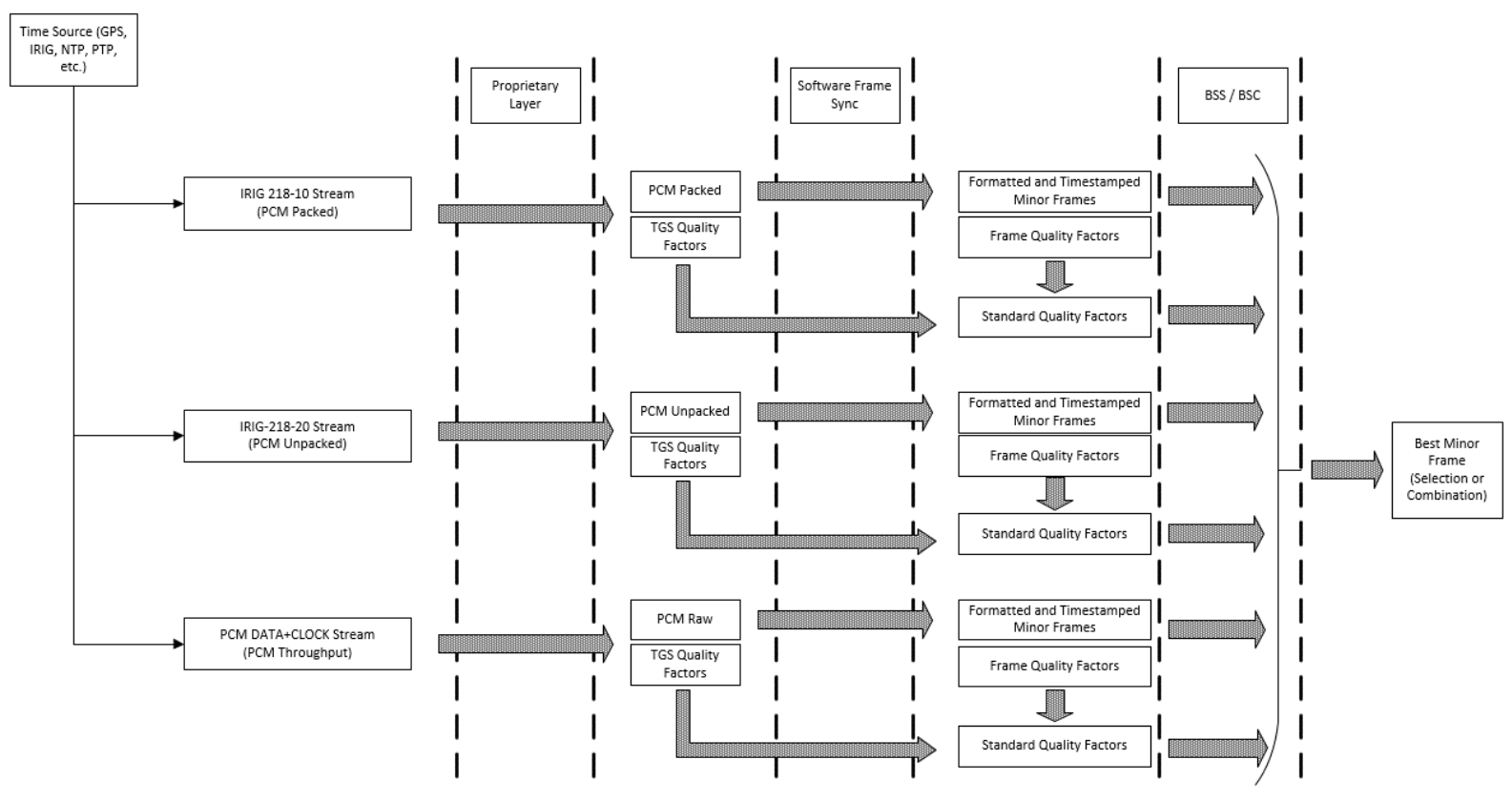

Figure 15: PCM Stream comparison/combination

In this example, the three sources are carrying the same PCM stream but in heterogeneous format. Proprietary layer extracts the PCM stream information and the TGS quality factors. Software Frame Sync formats the PCM streams in comparable data frames, extract frame quality factors so it is possible to compare it in the BSS. All the minor frames are timestamped and can be compared bit to bit with the standardized weighted quality factors.

\section{Packet Synchronization}

The main challenge we had to deal with was the packet synchronization: Ethernet protocols are buffered and non-deterministic. The propagation time of the telemetry signals is dependent on the varying distance between the mobile and the antenna, thus causing varying delays. If there is no information available on the PCM data to timestamp and evaluate this delay, BSS/BSC architecture could produce invalid results if the delay is greater than a long cycle duration.

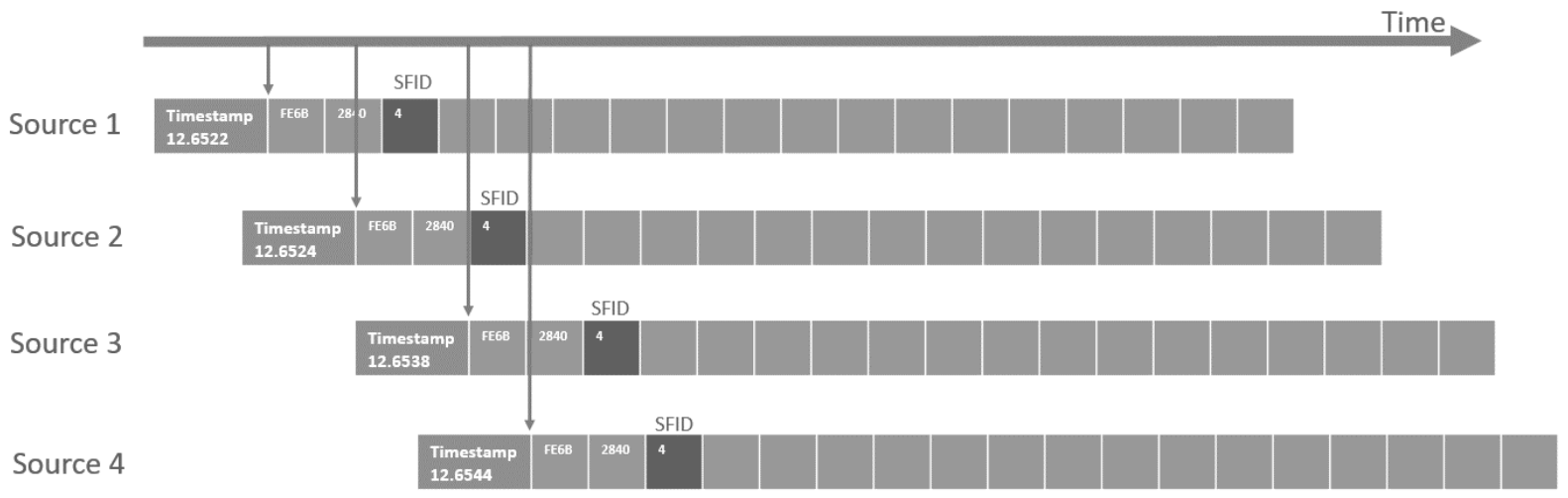

Figure 16: Example of receiving time desynchronization

Most of telemetry systems timestamp the data at receiving time, PCM frames must include some identification parameters (SFID, counters, etc.) to be able to synchronize and compare the short cycles. Figure below presents an example of synchronization using timestamps and SFID values. 


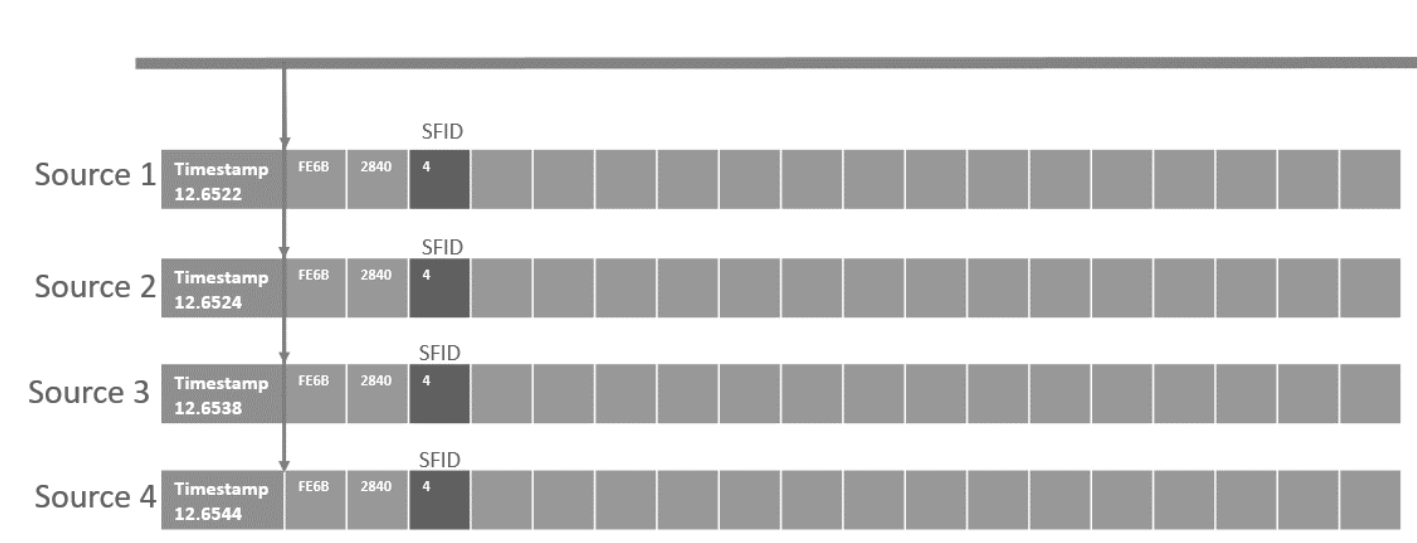

Figure 17: Example of synchronization algorithm using timestamps and SFID values

\section{Practical case}

To validate this model, several architectures have been tested with good results.

The Three layers BSS have been implemented in MAGALI Telemetry Software used as the telemetry data software.

The first practical case is based on Telemetry receivers sending proprietary Ethernet Output in throughput mode which are connected to MAGALI decom station. Each Telemetry Receiver sends three Ethernet Outputs (LHCP / RHCP / Combined) at $30 \mathrm{Mbps}$ to the MAGALI telemetry software.

The decom station had to produce a QLM (Quick Look Message) stream. This is an Ethernet message $(50 \mathrm{~Hz})$ composed of selected Telemetry parameters (filtered from ICD).

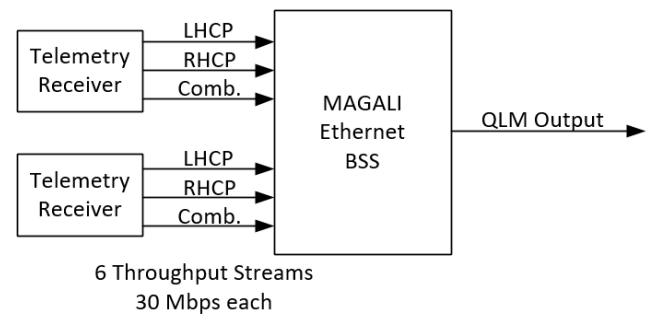

Figure 18: 30Mbps streams / QLM Output

The Telemetry Stream is a 30Mbps PCM-FM with $50 \mu$ s minor frames.

The tests have been done on Core i7-6700@3.4GHz CPU. CPU Load was around 20\% for the six streams with a $130 \mathrm{Mbps}$ Ethernet Load. Delay measurement was around 60ms with a deviation of 30 ms.

Tests have shown that this solution ensures signal continuity over long distances by defining the best time to switch from one source to another automatically.

The second practical case is based on three heterogeneous sources as shown in figure below. The system had to generate the best Ch10 unpacked telemetry stream. The sources came from several installation on the range.

- A new receiver able to provide TM throughput signals in Ch10 format

- A MAGALI TGS front end which provides Frame synchronized data blocks

- A PCM source which has been upgraded few years ago with a PCM to Ethernet Module to broadcast telemetry over Ethernet. This source was a redundant source in case of failure as there is no other quality factor than Frame sync Lock. 


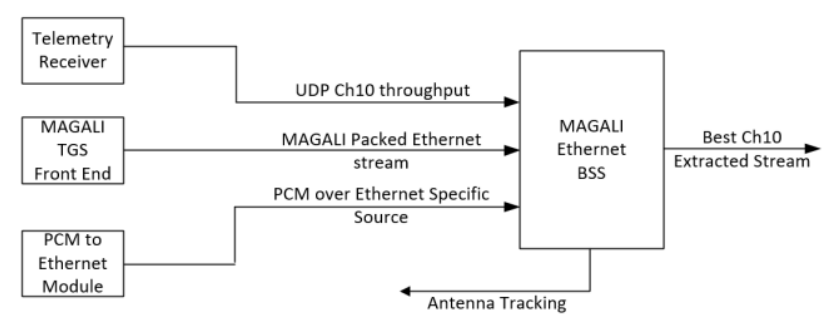

Figure 19: Heterogeneous sources

The bit rate in this case does not exceed 10Mbits but the interesting aspect is the heterogeneous sources as PCM inputs. Even if the complete installation has not been upgraded with the same hardware interfaces, the Ethernet BSS is able to manage the different kind of inputs. Therefore, end customer did not have to buy other hardware to manage the different inputs. Time delay has not been measured for this project, nevertheless the system was performant and delay was not exceeding $200 \mathrm{~ms}$ to provide the Ch10 stream in real time.

The best source selection algorithm used in this case (with 3 systems) allows to correct the frames and obtain $42 \%$ of corrected bits compared to a single-stream system ( $20 \%$ in term of corrected frames).

The third practical case is based on four heterogeneous sources as shown in figure below. The sources came from: two receivers sending TMoIP (IRIG 218-10) streams and a MAGALI TGS front end which provides 2 frames of synchronized data blocks.

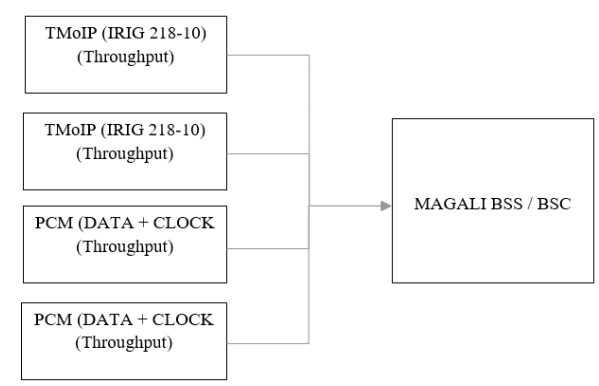

Figure 20: TMoIP and PCM DATA+CLOCK

The bit rate in this case is $20 \mathrm{Mbits}$ but the interesting aspect is the heterogeneous sources as PCM inputs and the heterogeneous format of quality factors (DQM for TMoIP, and frame quality factors for PCM DATA+CLOCK). The MAGALI system had to compute standardized quality values to permit the BSS/BSC module to perform selection and/or combination.

The combination algorithm used in this case (with 4 systems) allows to correct the frames and obtain

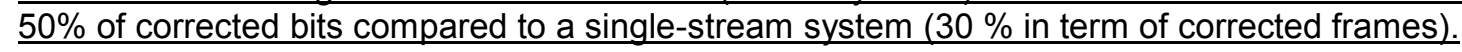

\section{Conclusion}

The BSS/BSC architecture is a good answer to:

- The prolific Ethernet PCM format that all the manufacturers can propose.

- The management of heterogeneous sources in case of a partial update of an existing installation.

This solution is a low cost investment as it is only software based. Moreover, the three layers architecture provides a flexible model in case of new format management, only the first layer has to be upgraded with a new plugin in order to manage the new format. Software Frame Sync \& BSS Layer remain the same once the information are provided.

The BSS/BSC algorithms allows to correct the frames and obtain from $42 \%$ to $50 \%$ of corrected bits compared to a single-stream system (20\% to $30 \%$ in term of corrected frames).

The constraint of this architecture is that the packets are timestamped using different formats and packets can be sent with a variable delay (as an example, Ethernet is a non-deterministic protocol). The BSS/BSC architecture must ensure that:

- The packets must be timestamped using a standard format. 
- The packet synchronization: the BSS must compare (and then combine or select) full synchronized packets using timestamps and counters extracted from the frames (SFID for example).

Another constraint of this architecture is in case of encrypted data, throughput data cannot be compared with synchronized data. It is necessary to decrypt all the data before using this method or to compare only encrypted throughput data based on a random sync pattern.

If all the streams are encrypted using the same algorithm, another solution would be to transmit these streams to the BSS / BSC module. In this case, a bit correlation could be applied in order to synchronize the frames. This mechanism, which consumes a lot of processor time, could be transferred to external processors (GPU for example)

Acknowledgement:

Cyril Lambert

NEXEYA, Test Range Skill Centre

Jean Guy PIEROZAK NEXEYA, Business Line Manager - Test Range Activities

Sabine LASSERRE NEXEYA, Marketing \& Quality

Julien MALIVERNEYi

\section{References:}

[1] ITC_2004_04-09-01: Telemetry Best Source Selection at White Sands Missile Range

[2] ITC_2004_04-23-01: Performance evaluation of Ethernet LAN based distributed telemetry data network and its extension using router and bridge

[3] ITC_2012_12-05-02: IRIG 106 Chapter 10 vs. iNET Packetization: Data Storage and Retrieval

[4] ITC_2012_12-24-01: PCM to Ethernet: A Hybrid System Used to Certify the Next Generation of Data Transfer Technology

[5] ITC_2018_18-09-03: BSS with Heterogeneous Ethernet Sources

i In memory of Julien MALIVERNEY (1978-2019), rest in peace my friend. 